\title{
Low expression of microRNA-30c promotes prostate cancer cells invasion involved in downregulation of KRAS protein
}

\author{
JUN ZHANG, XILONG WANG, YANGYUN WANG, RUIXIAN PENG, ZHIYUAN LIN, \\ YANG WANG, BO HU, JIFENG WANG and GUOWEI SHI \\ Department of Urology, The Fifth People's Hospital of Shanghai, Fudan University, Shanghai 200240, P.R. China
}

Received February 18, 2016; Accepted March 3, 2017

DOI: $10.3892 / \mathrm{ol} .2017 .6163$

\begin{abstract}
Aberrant microRNA expression is associated with tumor development. The present study aimed to elucidate the role of miR-30c in the development of prostate cancer. Quantitative polymerase chain reaction was performed to compare miR-30c expression in LNCaP, DU145, PC-3 and RWPE-1 cell lines. Lentivirus expressing miR-30c was used to create stable overexpression cell lines to investigate the effects of miR-30c overexpression on cell proliferation, migration and invasion, which were determined in the prostate cancer cell line PC-3 by MTT, colony formation, wound healing and Transwell assays. Effects of miR-30c on KRAS were examined by western blot analysis. miR-30c expression was significantly lower $(\mathrm{P}<0.05)$ in the $\mathrm{PC}-3$ cell line compared with LNCaP, DU145 and RWPE-1 cell lines. miR-30c overexpression in PC-3 inhibited tumor cell proliferation, migration and invasion in vitro. Furthermore, KRAS protein expression was downregulated in miR-30c overexpression cell lines compared with the negative control $(\mathrm{NC})$ group $(\mathrm{P}<0.05)$. The present results demonstrated that overexpression of miR-30c inhibits prostate cancer cell line proliferation, migration and invasion, which was possibly caused by downregulation of KRAS protein by miR-30c. The data implicate miR-30c in the prognosis and treatment of prostate cancer.
\end{abstract}

\section{Introduction}

Prostate cancer is one of the most common malignant tumors in the urinary and reproductive system (1-3). Prostate cancer is the second most common cancer globally and has the sixth highest mortality rate (4). In previous years, microRNA (miRNA) regulation and its role in the occurrence and development of prostate cancer has become a focus of study.

Correspondence to: Dr Guowei Shi, Department of Urology, The Fifth People's Hospital of Shanghai, Fudan University, 801 Heqing Road, Minhang, Shanghai 200240, P.R. China

E-mail: drsgw@189.cn

Key words: microRNA-30c, prostate cancer, KRAS, invasion
miRNAs are 21-25 nucleotide non-coding, single-stranded RNA, which regulate gene expression at the transcriptional and translational level. miRNAs may regulate tumor occurrence, development and prognosis through the oncogenes or tumor suppressor genes (5). Due to the different expression of miRNAs in normal tissues and malignant tissues, the expression profile of miRNAs in different tissues may be a potential tool for the diagnosis, targeted therapy and prognosis of tumors. In 2007, Porkka et al (6) used gene chip technology for the first time to report the different expression pattern of miRNAs in benign and malignant prostate tumors. Ambs et al (7) studied the differences in miRNA and mRNA in 60 cases of prostate cancer and normal prostate tissue and revealed that miRNA is associated with the development of prostate cancer, and miRNA controls tumor growth by regulating the expression of tumor-associated genes to promote prostate cancer occurrence and development. Chen et al (8) and other comparative studies $(9,10)$ of the expression of miRNA in the plasma of patients with prostate cancer and benign prostatic hyperplasia revealed that the expression of miR-622 and miR-1285 significantly increased, while the expression of let-7e, Let-7c and miR-30c significantly decreased. Therefore, the present study hypothesized that the differential expression of the 5 miRNAs in the plasma may be used as a biochemical marker and, combined with the prostate-specific antigen test, can be used in the diagnosis of prostate cancer. Sylvestre et al (11) and other studies (12) demonstrated that miR-20a is overexpressed in prostate cancer, and overexpression of miR-20a in PC-3 cells exhibits an anti-apoptotic effect. Qin et al (13) reported that miR-24 regulates the expression of the target gene fas associated factor 1, and induces the apoptosis of hormone refractory prostate cancer cells. Bin et al confirmed that miR-146a (14) inhibits the progression of prostate cancer tumors through the epidermal growth factor receptor signaling pathway.

Previous studies have demonstrated that $\mathrm{miR}-30 \mathrm{c}$ is expressed in numerous types of malignant tumors, including breast, ovary, stomach and prostate cancers. Lee et al (15) previously reported that mir-30c had significantly lower expression in ovarian cancer tissue compared with normal ovarian tissue, and additional multivariate analysis revealed that patients with ovarian cancer mir-30c high expression had longer survival and overall survival rate. Zhou et al (16) revealed that miR-30c is a tumor suppressor gene that can inhibit the growth of endometrial cancer by targeting the expression of metastasis-associated 
1. In lung cancers, Zhong et al demonstrated that low expression of miR-30c promotes invasion by inducing epithelial mesenchymal transition (17). Wu et al (18) identified that miR-30c overexpression inhibits the migration and invasion of the hepatocellular carcinoma cell lines SMMC-7721 and HepG2. Therefore, overexpression of miR-30c may inhibit the proliferation, migration and invasion of tumor cells. However, Rodríguez-González et al (19) reported that high expression of miR-30c may be used as an independent predictor of the efficacy of endocrine therapy for breast cancer and tumor progression free survival. Dobson et al (20) demonstrated that overexpression of miR-30c in the breast cancer MDA-MB-231 cell line promotes the invasive metastatic phenotype. Therefore, the role of miR-30c in different tumors remains controversial.

Previous studies have reported that plasma mir-30c exhibited low expression in prostate cancer tissue $(6,8)$. However, the mechanism of miR-30c involvement in the development and progression of prostate cancer remains unclear, and the target gene is not clear. The present study aimed to examine the underlying mechanism of the association between miR-30c and prostate cancer growth in order to provide additional evidence to facilitate the improvement of therapeutic strategies for this disease.

\section{Materials and methods}

Cell culture. LNCaP, DU145, PC-3 and RWPE-1 (American Type Culture Collection, Manassas, VA, USA) were cultured in Roswell Park Memorial Institute (RPMI)-1640 medium with $10 \%$ fetal bovine serum (FBS; Invitrogen; Thermo Fisher Scientific, Inc., Waltham, MA, USA) and penicillin (100 U/ml). The cells were cultured at $37^{\circ} \mathrm{C}$ with $5 \% \mathrm{CO}_{2}$.

Plasmid construction and transfection. A miR-30c expression vector was constructed by cloning the fragment, amplified from human genomic DNA by polymerase chain reaction (PCR) with the primers, hmiR30c-EcoRI (forward, 5'-CCG GAATTCAACATAGTGTGGGGATGGGGT-3') and hmiR30c-BamHI (reverse, 5'-CGCGGATCCAGGTTAATG GGAAACAGGGCT-3') into the EcoRI and BamHI sites of the pLV-mCherry(2A)puro vector. The pLV-mCherry(2A) puro vector and packaging vector were co-transfected into HEK293T cells (American Type Culture Collection) to obtain high titer virus particles with replication defects. For lentiviral infection, cells were plated at a concentration of $1 \times 10^{5}$ cells/well onto 6-well culture plates and then infected with indicated lentiviruses in the presence of $8 \mu \mathrm{g} / \mathrm{ml}$ polybrene. Following infection for $72 \mathrm{~h}$, puromycin was added to the medium at a concentration of $0.5 \mu \mathrm{g} / \mathrm{ml}$ for PC-3 cells and cell populations were selected for 2 weeks for following experiments.

Isolation of total RNA and quantitative PCR (qPCR). Total RNA was extracted from collected cells using TRIzol reagent (Invitrogen; Thermo Fisher Scientific, Inc.) and mRNA was then reverse transcribed to cDNA (Promega Corporation, Madison, WI, USA). U6 small nuclear RNA was used for normalization. The PCR reactions were performed with the following primers: hsa-miR-30c forward, 5'-CCCGCTGTA AACATCCTACACTC-3' and reverse, 5'-GTGCAGGGT CCGAGGT-3'; and U6 forward, 5'-CGCTTCGGCAGCACA
TATAC-3' and reverse, 5'-CAGGGGCCATGCTAATCTT-3'. qPCR was performed using the ABI 7900 Fast Real-Time PCR system (Applied Biosystems; Thermo Fisher Scientific, Inc.).

Cell cycle analysis. The cell cycle was assessed at $24 \mathrm{~h}$ following transfection by propidium iodide staining, and measured with a FACS Calibur flow cytometer (BD Biosciences, San Jose, CA, USA). MTT PC-3 cells were trypsinized and seeded onto 96-well plates at a density of $3 \times 10^{3}$ cells/well for culture. Cell proliferation was measured using the MTT assay 24, 48 and $72 \mathrm{~h}$ after seeding. Briefly, $10 \mu \mathrm{l}$ of MTT solution $(5 \mathrm{mg} / \mathrm{ml})$ was added to each well, and the cells were incubated for $3 \mathrm{~h}$ at $37^{\circ} \mathrm{C}$. The medium was then aspirated, and $150 \mu \mathrm{l}$ of dimethyl sulfoxide was added. The optical density was measured at $570 \mathrm{~nm}$ with a microplate reader (Bio-Rad Laboratories, Inc., Hercules, CA, USA). Three independent experiments were performed in quadruplicate. The cell growth inhibition rate $(\%)=(1-$ value of experimental group/negative control group) x100.

Colony formation assay. The two cells types were harvested and 1,000 cells were inoculated in each pore of a 6-well plate. The medium was changed every 2 days. After 14 days, cells were rinsed with 1X PBS and then fixed with methanol for $10 \mathrm{~min}$. Subsequently, cells were stained with $0.1 \%$ crystal violet (Sigma-Aldrich; Merck KGaA, Darmstadt, Germany) for $>1$ h.

Wound healing assay. The cells were seeded onto 6-well plates and cultured with RPMI-1640 medium. Following $24 \mathrm{~h}$, the cells were wounded with a $200 \mu$ l pipette tip. Serum-free RPMI-1640 medium was added and wound closure was observed for $30 \mathrm{~h}$ using an XSP-4C microscope at a magnification of x200 (Shanghai Changfang Optical Instrument Co. Ltd., Shanghai, China).

Transwell assay. Cell motility was measured using an $8 \mu \mathrm{m}$ pore polycarbonate membrane Boyden chamber insert in a Transwell apparatus (EMD Millipore, Billerica, MA, USA). The transfected cells were treated with $0.2 \%$ trypsin/EDTA solution and washed once with serum-containing RPMI-1640 medium. A total of $1.5 \times 10^{5}$ cells in $0.2 \mathrm{ml}$ serum-free RPMI-1640 medium were seeded onto a Transwell apparatus. RPMI-1640 medium containing $10 \%$ FBS $(600 \mu \mathrm{l})$ was added to the lower chamber. An invasion assay was conducted following the aforementioned procedure, with the exception that the filters of the Transwell chambers were coated with $45 \mu \mathrm{g}$ Matrigel (BD Biosciences). Following incubation of the cells for 24,48 and $72 \mathrm{~h}$ at $37^{\circ} \mathrm{C}$ in a $5 \% \mathrm{CO}_{2}$ incubator, the cells on the top surface of the insert were removed by wiping with a cotton swab. The cells that invaded to the bottom surface of the insert were fixed in the $100 \%$ precooling methanol for $10 \mathrm{~min}$, stained with $0.1 \%$ crystal violet for $30 \mathrm{~min}$, rinsed in PBS and then subjected to microscopic inspection (XSP-4C microscope; magnification, $\mathrm{x} 200$ ). The values for invasion were obtained by counting three fields per membrane and were represented as the average of the three independent experiments.

Western blot analysis. The total proteins were prepared from the established cells. The proteins were fractionated by SDS-PAGE, transferred to a polyvinylidene fluoride membrane (EMD Millipore), blocked in 5\% dry milk at room temperature 
for $1 \mathrm{~h}$ and immunostained with antibodies at $4^{\circ} \mathrm{C}$ overnight using anti-KRAS (dilution, 1:1,000; \#sc-30; Santa Cruz Biotechnology, Inc., Dallas, TX, USA) and anti-GAPDH (dilution, 1:5,000; \#sc-47724; Santa Cruz Biotechnology, Inc.). All results were visualized through an electrochemiluminescence substrate western blotting detection system (Pierce; Thermo Fisher Scientific, Inc.) and then exposed by the Molecular Imager ChemiDoc XRS System (Bio-Rad Laboratories, Inc.). The integrated density of the band was quantified by ImageJ software v1.37 (Bio-Rad Laboratories, Inc.).

Statistical analysis. The $2^{-\Delta \Delta \mathrm{Cq}}$ method was used to analyze the results of qPCR in all the experiments performed in the present study (21). Statistical analysis was performed using SPSS 10.0 (SPSS, Inc., Chicago, IL, USA) and presented with GraphPad prism software v6.01 (GraphPad Software, Inc., La Jolla, CA, USA). The results obtained from experiment in vitro assays are presented as the mean \pm standard deviation from at least 3 independent experiments, and the data were analyzed by the $\mathrm{t}$-test. $\mathrm{P}<0.05$ was considered to indicate a statistically significant difference.

\section{Results}

Expression of $\mathrm{miR}-30 \mathrm{c}$ in the PC-3 cell line is lower than other human prostate cell lines. It was reported that the expression level of $\mathrm{miR}-30 \mathrm{c}$ in prostate cancer tissue and plasma was low (22). In order to study the function of miR-30c in the prostate cell lines, the expression of miR-30c in human prostate cell lines was tested by reverse transcription-qPCR. PC-3 was revealed to possess significantly lower miR-30c expression than the LNCaP, DU145 and RWPE-1 cell lines $(\mathrm{P}<0.001$; Fig. 1). Therefore, based on this expression pattern, the PC-3 cell line was selected to verify the effect of miR-30c.

miR-30c regulates the cell cycle of $\mathrm{PC}-3$ cells in vitro. To examine the mechanism underlying the effect of miR-30c on tumor growth in prostate cancer, the PC-3 cells were transfected with control miRNA (NC) and miR-30c. The transfection efficiency was validated by qPCR. The cell cycle assay performed by fluorescence correlation spectroscopy demonstrated that overexpression of miR-30c caused the accumulation of PC-3 cells in the G2/M phase compared with the $\mathrm{NC}(\mathrm{P}<0.05$; Fig. 2). The results indicated that miR-30c may inhibit growth of the PC-3 cell line in vitro.

miR-30c overexpression inhibits the growth of the PC-3 cell line. To verify whether miR-30c regulates tumor growth in prostatic cancer, the MTT test was used to study PC-3 cell growth, which had been transfected with miR-30c NC or miR-30c. The transfection efficiency was validated by $\mathrm{qPCR}$. Overexpression of miR-30c significantly inhibited the growth of PC-3 cells compared with the control at 48 and $72 \mathrm{~h}(\mathrm{P}<0.05$; Fig. 3). The present results indicated that miR-30c inhibits the growth of the PC-3 cell line in vitro.

Role of miR-30c overexpression in the PC-3 cell line colony formation ability. To assess whether the overexpression of miR-30c expression affected the tumorigenic properties of the cells, the colony formation assay was used (Fig. 4).

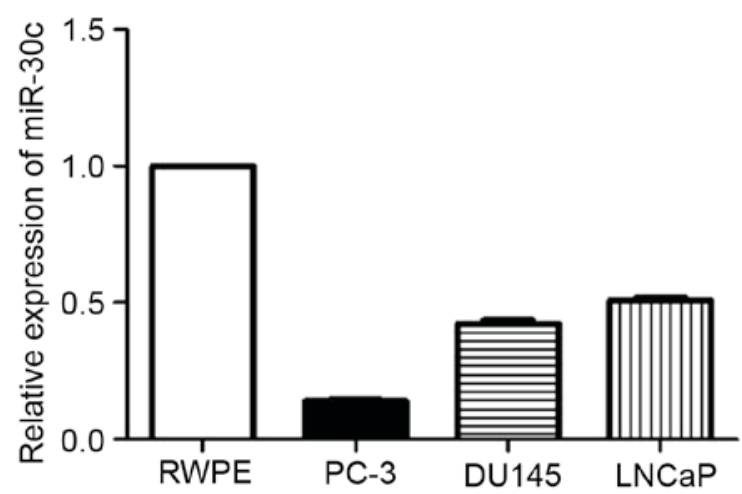

Figure 1. miR-30c expression is lower in the PC-3 cell line compared with other prostate cancer cell lines. Expression levels of miR-30c in prostatic cancer cell lines were determined by quantitative polymerase chain reaction. PC-3 cells compared with RWPE, DU145 and LNCaP cells.

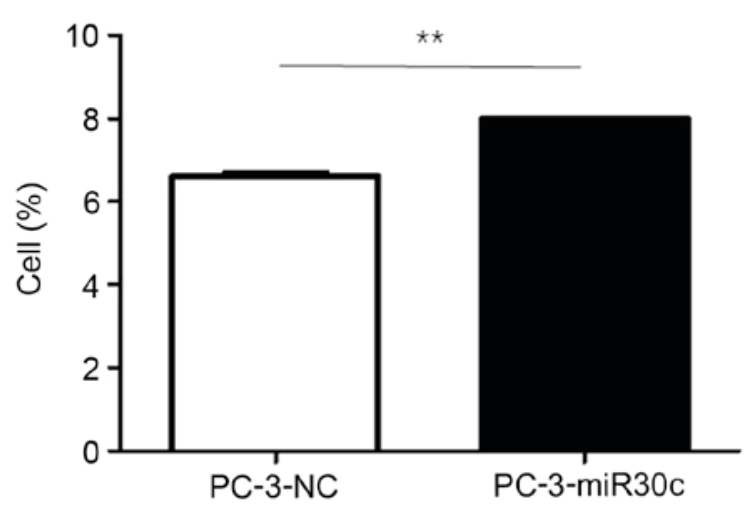

Figure 2. miR-30c negatively regulates the PC-3 cell line cell cycle. $G_{2} / M$ phase cells in miR-30c overexpression cells (PC-3-miR30c) compared with $\mathrm{NC}$ overexpression cells (PC-3-NC). ${ }^{* *} \mathrm{P}<0.001$. NC, control miRNA.

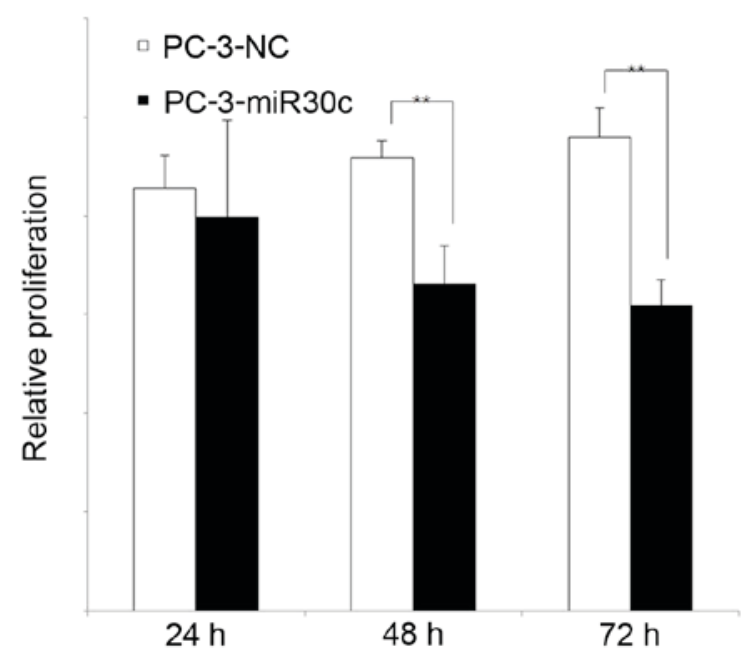

Figure 3. miR-30c inhibits PC-3 cell growth. Effects of miR-30c on the proliferation of PC-3 cell lines were analyzed by MTT at 24, 48 and $72 \mathrm{~h}$. ${ }^{* *} \mathrm{P}<0.001$. NC, control miRNA.

Following inoculation for 14 days, miR-30c transfected cells exhibited a significantly lower colony formation ability compared with the control cells. Therefore, it was concluded 
A

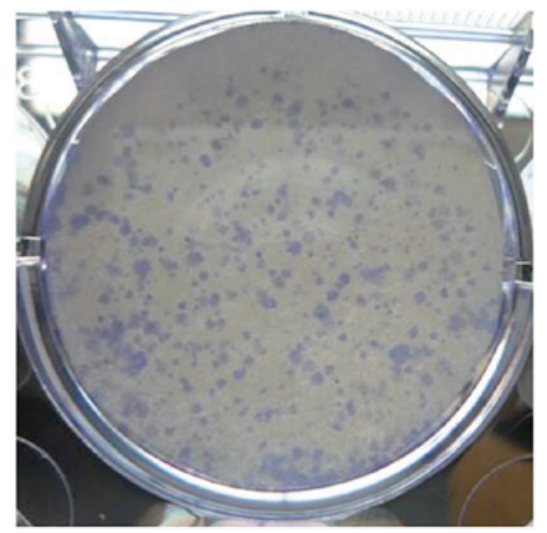

B

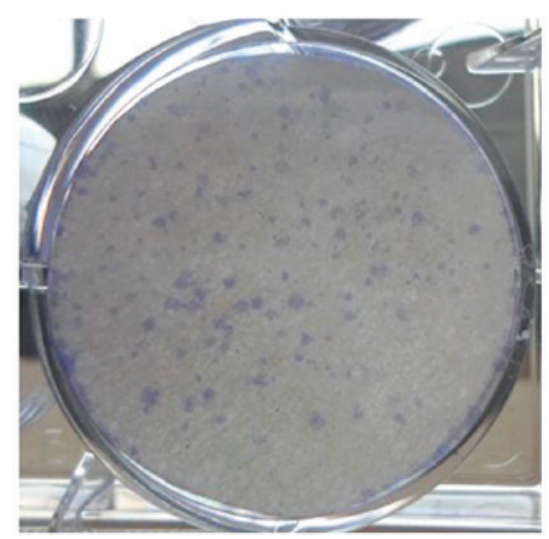

Figure 4. Overexpression of miR-30c inhibits the colony forming ability of the PC-3 cell line. (A) Colony formation assay of PC-3 NC cells at 14 days following inoculation. (B) Colony formation assay of PC-3 miR-30c cells at 14 days following inoculation. NC, control miRNA.

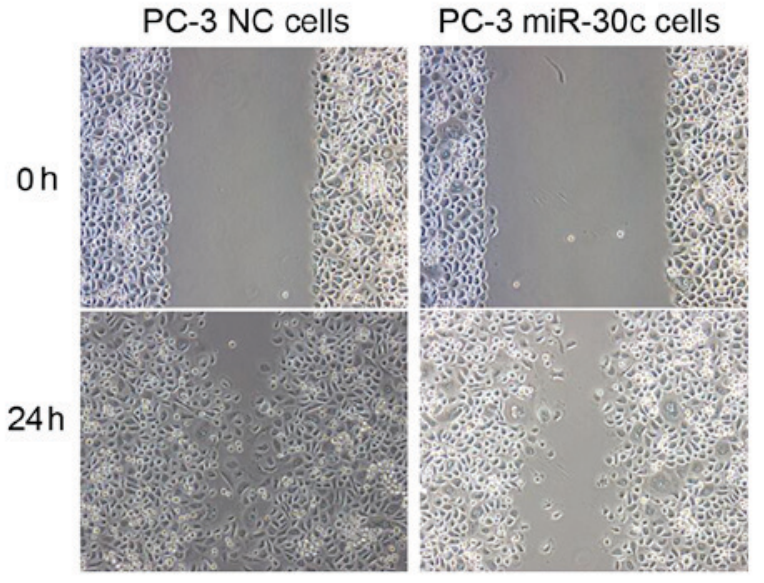

Figure 5. Effects of miR-30c expression on PC-3 cell wound healing. Images were captured at 0 and $24 \mathrm{~h}$ post-wounding (magnification, $\mathrm{x} 200$ ).

that miR-30c contributed to the regulation of PC-3 cell line growth.

$m i R-30$ c regulates the invasion of $P C-3$ cells in vitro. To examine whether miR-30c affects tumor cell invasion, the PC-3 cells were transfected with miR-30c and NC. The transfection efficiency was validated by qPCR. The wound healing assay demonstrated that the overexpression of miR-30c was able to suppress PC-3 cell healing (Fig. 5). Furthermore, the Transwell assay demonstrated that overexpression of miR-30c attenuated PC-3 cell invasion (Fig. 6). These results indicated that overexpression of miR-30c may inhibit invasion of the PC-3 cell line in vitro.

Overexpression of miR-30c is associated with the downregulation of KRAS expression. It was previously reported that KRAS is a target of miR-30c in breast cancer (23). The present study aimed to investigate whether miR-30c can control KRAS in prostatic cancer. Using western blot analysis, overexpression of miR-30c was revealed to significantly inhibit the expression of KRAS protein compared with the control (Fig. 7). The
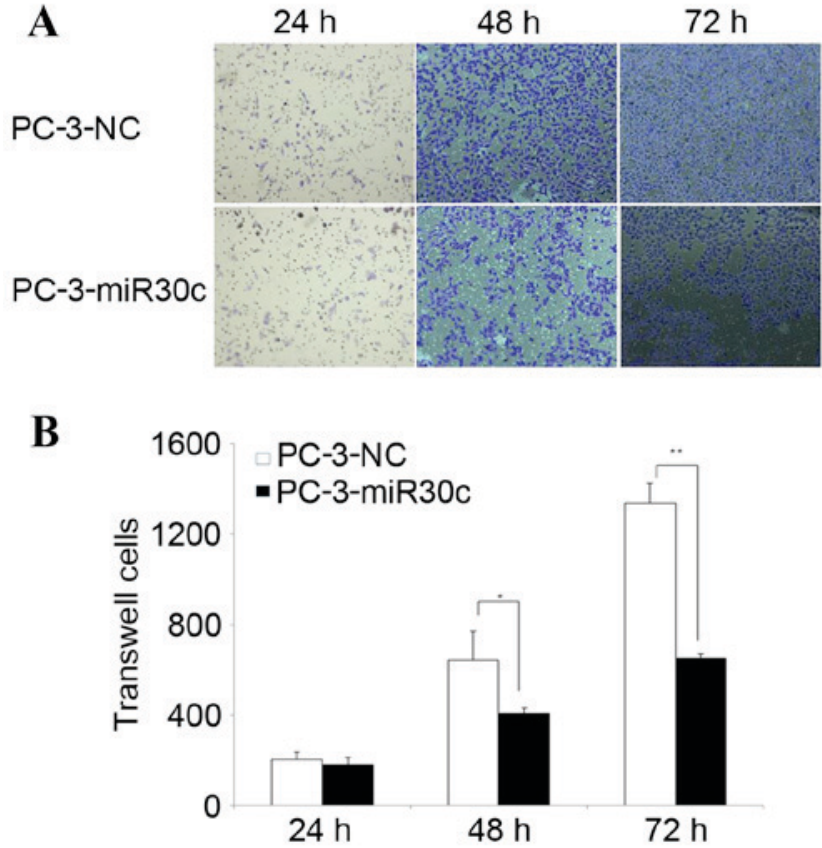

Figure 6. Effects of miR-30c expression on PC-3 cell invasion. The Transwell assay was performed as described in the methods. (A) The representative images of invasive cells at the bottom of the membrane stained with crystal violet were visualized at 24,48 and $72 \mathrm{~h}$. (B) Statistical analysis of Transwell assay data at 24,48 and $72 \mathrm{~h} .{ }^{*} \mathrm{P}<0.05 ;{ }^{* *} \mathrm{P}<0.001$. Magnification, $\mathrm{x} 200$.

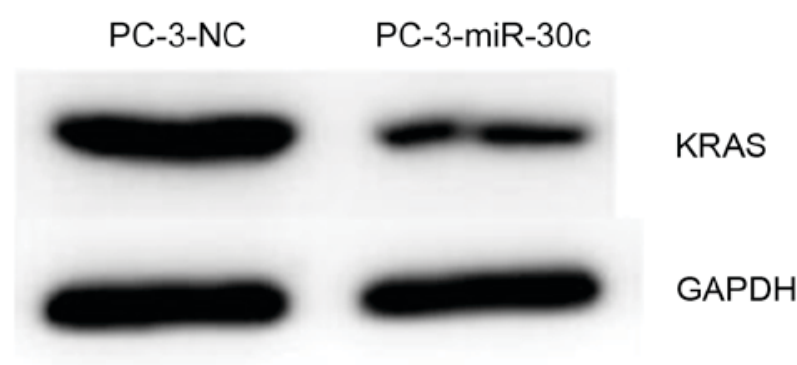

Figure 7. miR-30c expression controls the expression levels of the tumor suppressor gene KRAS. Western blot analysis of KRAS protein in miR-30c expression and control miRNA PC-3 cell line, using GAPDH as a control. 
present data indicated that there may be a common underlying mechanism of tumor progression.

\section{Discussion}

In the present study, it was demonstrated that overexpression of miR-30c inhibits prostate cancer cell line proliferation, migration and invasion, which is possibly involved in downregulation of KRAS protein. Thus, the data provided support a mechanism by which miR-30c serves a tumor suppressive role in prostate cancer.

It was previously reported $(6,8)$ that plasma mir-30c exhibits low expression in prostate cancer tissue. At present, however, the mechanism of miR-30c involvement in the development and progression of prostate cancer remains unclear, and the target gene is also uncertain. The present study aimed to examine the underlying mechanism of the association between miR-30c and prostate cancer growth, in order to provide additional evidence to facilitate improvement of the therapeutic strategies for this disease.

miRNA expression profiles are distinct among different tumor types and often reflect the tissue of origin and differentiation state of the tumor $(24,25)$. Previous studies have shown that miR-30c is overexpressed in breast cancer $(26,18)$. By contrast, miR-30c has low expression in prostate cancer tissue and plasma. The role of miR-30c involved in the development and progression of prostate cancer is not clear, and the target gene is not clear. In the present study, it was confirmed that miR-30c expression is lower in the PC-3 cell line compared with other prostate cancer cell lines; therefore, the PC-3 cell line was used to study the function of miR-30c. To investigate the role of miR-30c in prostate cancer, PC-3 cell lines overexpressing miR-30c were used. Reduced tumor proliferation and cell cycle block was identified in miR-30c overexpression PC-3 cells compared with negative control miRNA overexpression PC-3 cells, which indicated that the miR-30c suppresses tumor metastasis.

It was previously reported that miR-30c can regulate KRAS in breast cancer (23), and the present study revealed that KRAS was downregulated in miR-30c overexpression cells compared with control cells. This indicated that KRAS may be a common target of miR-30c in different types of tumors.

In conclusion, the present study demonstrated that overexpression of miR-30c inhibits prostate cancer cell proliferation, migration and invasion. miR-30c-mediated inhibition of PC-3 invasive phenotypes may be caused by the downregulation of KRAS protein.

\section{Acknowledgements}

The authors acknowledge financial support from the Health and Family Planning Commission Foundation of Shanghai Minhang District (grant no. 2013MW05) and the Shanghai Key Medical Specialty Program (grant no. ZK2015B04).

\section{References}

1. Peyromaure EM, Mao K, Sun Y, Xia S, Jiang N, Zhang S, Wang G, Liu Z and Debré B: A comparative study of prostate cancer detection and management in China and in France. Can J Urol 16: 4472-4477, 2009.
2. Zeigler-Johnson CM, Rennert H, Mittal RD, Jalloh M, Sachdeva R, Malkowicz SB, Mandhani A, Mittal B, Gueye SM and Rebbeck TR: Evaluation of prostate cancer characteristics in four populations worldwide. Can J Urol 15: 4056-4064, 2008.

3. Torre LA, Siegel RL, Ward EM and Jemal A: Global cancer incidence and mortality rates and Trends-An update. Cancer Epidemiol Biomarkers Prev 25: 16-27, 2016.

4. Jemal A, Bray F, Center MM, Ferlay J, Ward E and Forman D: Global cancer statistics. CA Cancer J Clin 61: 69-90, 2011.

5. Shi XB, Tepper CG and deVere White RW: Cancerous miRNAs and their regulation. Cell Cycle 7: 1529-1538, 2008.

6. Porkka KP, Pfeiffer MJ, Waltering KK, Vessella RL, Tammela TL and Visakorpi T: MicroRNA expression profiling in prostate cancer. Cancer Res 67: 6130-6135, 2007.

7. Ambs S, Prueitt RL, Yi M, Hudson RS, Howe TM, Petrocca F, Wallace TA, Liu CG, Volinia S, Calin GA, et al: Genomic profiling of microRNA and messenger RNA reveals deregulated microRNA expression in prostate cancer. Cancer Res 68: 6162-6170, 2008

8. Chen ZH, Zhang GL, Li HR, Luo JD, Li ZX, Chen GM and Yang J: A panel of five circulating microRNAs as potential biomarkers for prostate cancer. Prostate 72: 1443-1452, 2012.

9. Kachakova D, Mitkova A, Popov E, Popov I, Vlahova A, Dikov T, Christova S, Mitev V, Slavov C and Kaneva R: Combinations of serum prostate-specific antigen and plasma expression levels of let-7c, miR-30c, miR-141 and miR-375 as potential better diagnostic biomarkers for prostate cancer. DNA Cell Biol 34: 189-200, 2015.

10. Cochetti G, Poli G, Guelfi G, Boni A, Egidi MG and Mearini E: Different levels of serum microRNAs in prostate cancer and benign prostatic hyperplasia: Evaluation of potential diagnostic and prognostic role. Onco Targets Ther 9: 7545-7553, 2016.

11. Sylvestre Y, De Guire V, Querido E, Mukhopadhyay UK, Bourdeau V, Major F, Ferbeyre G and Chartrand P: An E2F/miR-20a autoregulatory feedback loop. J Biol Chem 282: 2135-2143, 2007.

12. Dhar S, Kumar A, Rimando AM, Zhang X and Levenson AS: Resveratrol and pterostilbene epigenetically restore PTEN expression by targeting oncomiRs of the miR-17 family in prostate cancer. Oncotarget 6: 27214-27226, 2015.

13. Qin W, Shi Y, Zhao B, Yao C, Jin L, Ma J and Jin Y: miR-24 regulates apoptosis by targeting the open reading frame $(\mathrm{ORF})$ region of FAF1 in cancer cells. PLoS One 5: e9429, 2010.

14. Xu B, Wang N, Wang X, Tong N, Shao N, Tao J, Li P, Niu X, Feng N, Zhang L, et al: MiR-146a suppresses tumor growth and progression by targeting EGFR pathway and in a p-ERK-dependent manner in castration-resistant prostate cancer. Prostate 72 : 1171-1178, 2012.

15. Lee H, Park CS, Deftereos G, Morihara J, Stern JE, Hawes SE, Swisher E, Kiviat NB and Feng Q: MicroRNA expression in ovarian carcinoma and its correlation with clinicopathological features. World J Surg Oncol 10: 174, 2012.

16. Zhou H, Xu X, Xun Q, Yu D, Ling J, Guo F, Yan Y, Shi J and $\mathrm{Hu}$ Y: microRNA-30c negatively regulates endometrial cancer cells by targeting metastasis-associated gene-1. Oncol Rep 27: 807-812, 2012.

17. Zhong Z, Xia Y, Wang P, Liu B and Chen Y: Low expression of microRNA-30c promotes invasion by inducing epithelial mesenchymal transition in non-small cell lung cancer. Mol Med Rep 10: 2575-2579, 2014.

18. Wu W, Zhang X, Liao Y, Zhang W, Cheng H, Deng Z, Shen J, Yuan Q, Zhang Y and Shen W: miR-30c negatively regulates the migration and invasion by targeting the immediate early response protein 2 in SMMC-7721 and HepG2 cells. Am J Cancer Res 5: 1435-1446, 2015.

19. Rodríguez-González FG, Sieuwerts AM, Smid M, Look MP, Meijer-van Gelder ME, de Weerd V, Sleijfer S, Martens JW and Foekens JA: MicroRNA-30c expression level is an independent predictor of clinical benefit of endocrine therapy in advanced estrogen receptor positive breast cancer. Breast Cancer Res Treat 127: 43-51, 2011.

20. Dobson JR, Taipaleenmäki H, Hu YJ, Hong D, van Wijnen AJ, Stein JL, Stein GS, Lian JB and Pratap J: hsa-mir-30c promotes the invasive phenotype of metastatic breast cancer cells by targeting NOV/CCN3. Cancer Cell Int 14: 73, 2014.

21. Livak KJ and Schmittgen TD: Analysis of relative gene expression data using real-time quantitative PCR and the 2(-Delta Delta C(T)) Method. Methods 25: 402-408, 2001.

22. Chen ZH, Zhang GL, Li HR, Luo JD, Li ZX, Chen GM and Yang J: A panel of five circulating microRNAs as potential biomarkers for prostate cancer. Prostate 72: 1443-1452, 2012. 
23. Tanic M, Yanowsky K, Rodriguez-Antona C, Andrés R, Márquez-Rodas I, Osorio A, Benitez J and Martinez-Delgado B: Deregulated miRNAs in hereditary breast cancer revealed a role for miR-30c in regulating KRAS oncogene. PLoS One 7: e38847, 2012.

24. Jay C, Nemunaitis J, Chen P, Fulgham P and Tong AW: miRNA profiling for diagnosis and prognosis of human cancer. DNA Cell Biol 26: 293-300, 2007
25. Lu J, Getz G, Miska EA, Alvarez-Saavedra E, Lamb J, Peck D, Sweet-Cordero A, Ebert BL, Mak RH, Ferrando AA, et al: MicroRNA expression profiles classify human cancers. Nature 435: 834-838, 2005

26. Yen MC, Shih YC, Hsu YL, Lin ES, Lin YS, Tsai EM, Ho YW, Hou MF and Kuo PL: Isolinderalactone enhances the inhibition of SOCS3 on STAT3 activity by decreasing miR-30c in breast cancer. Oncol Rep 35: 1356-1364, 2016. 